\title{
STDP, Hebbian cell assemblies, and temporal coding by spike synchronization
}

\author{
Andreas Knoblauch ${ }^{1 *}$, Florian Hauser ${ }^{2}$ \\ From Twentieth Annual Computational Neuroscience Meeting: CNS*2011 \\ Stockholm, Sweden. 23-28 July 2011
}

The question whether neural activity follows a rate code or a temporal code is still an unsolved issue in neuroscience. Recent theoretical works have suggested that realistic models of spike-timing-dependent-plasticity (STDP) in recurrent networks could resolve this issue because they observed a seemingly unique relation between the neural code and the resulting pattern of synaptic connections [1]. In particular, these works argue that temporal coding would be inconsistent with bidirectional synaptic connectivity as observed in visual cortex [2] and expected for Hebbian cell assemblies ("what fires together wires together"). This conclusion is based on two assumptions, first, that simple STDP based on spike doublets would be generally unable to produce bidirectional synaptic connectivity (see references in [1]) and, second, that realistic voltage-based STDP would be able to produce bidirectional synaptic connectivity only for rate coding where signals are coded by cell ensembles jointly elevating firing rates on a time scale of hundreds of milliseconds [1].

We have disproved both assumptions. In particular, here we show that already simple STDP models can easily grow bidirectional connections for a temporal code based on spike synchronization unless synchronization is extremely precise (see [3] for disproving the second assumption). To this end we present simulation results for various STDP models [4] including all-to-all (AA) doublet STDP, nearest-neighbor (NN) doublet STDP, and various triplet STDP model variants with parameters fitted to experimental data and including realistic propagation delays. It turns out that all model variants can grow functional cell assemblies as reflected by strong bidirectional connectivity and tested within an associative memory framework [5]. This requires coarsely synchronized inputs such that spikes of

\footnotetext{
* Correspondence: andreas.knoblauch@honda-ri.de

${ }^{1}$ Honda Research Institute EU, 63073 Offenbach/Main, Germany

Full list of author information is available at the end of the article
}

the neurons within a cell assembly get synchronized on a time scale of 5 to 50 milliseconds, whereas more precise synchronization leads to depression of synaptic weights and, thus, destruction of the cell assembly. For NN doublet STDP and triplet STDP synaptic potentiation is boosted by increasing the rate of synchronized inputs, whereas high rates destroy cell assemblies for AA doublet STDP.

Thus, both rate coding and temporal coding based on coarse synaptic synchronization can account for the bidirectional connectivity observed, for example, in visual cortex [2]. However, we argue that the temporal code will be much more energy efficient for learning because it allows to grow and preserve cell assemblies at low mean firing rates at the level of spontaneous activity, whereas a rate code (based on uncorrelated Poissonian firing) can grow cell assemblies only by maintaining high firing rates over longer time intervals. Moreover, additional simulation experiments demonstrate how STDP can quickly grow multiple independent cell assemblies reflecting the spike timing relations of simultaneously active input neurons. We speculate that such a multiplexing mechanism may play a role both for working memory and long-range communication where "copies" or "indices" [6] of cell assemblies could easily be transmitted between cortical areas.

\section{Author details}

${ }^{1}$ Honda Research Institute EU, 63073 Offenbach/Main, Germany. ${ }^{2}$ Institute of Neural Information Processing, Ulm University, 89069 Ulm, Germany.

Published: 18 July 2011

\section{References}

1. Clopath C, Büsing L, Vasilaki E, Gerstner W: Connectivity reflects coding: a model of voltage-based STDP with homeostasis. Nature Neuroscience 2010, 13:344-352.

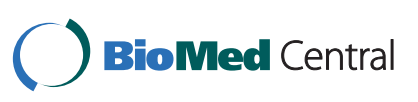

(c) 2011 Knoblauch and Hauser; licensee BioMed Central Ltd. This is an open access article distributed under the terms of the Creative Commons Attribution License (http://creativecommons.org/licenses/by/2.0), which permits unrestricted use, distribution, and reproduction in any medium, provided the original work is properly cited. 
2. Song S, Sjöström PJ, Reigl M, Nelson S, Chklovskii DB: Highly nonrandom features of synaptic connectivity in local cortical circuits. PLOS Biology 2005, 3:507-519.

3. Hauser F, Palm G, Knoblauch A: Different neural codes result in bidirectional connectivity formed by the same model of spike-timingdependent plasticity. BMC Neuroscience 2011, same supplement.

4. Morrison A, Diesmann M, Gerstner W: Phenomenological models of synaptic plasticity based on spike timing. Biological Cybernetics 2008, 98:459-478.

5. Knoblauch A, Palm G: Pattern separation and synchronization in spiking associative memories and visual areas. Neural Networks 2001, 14:763-780.

6. Teyler TJ, Rudy JW: The hippocampal indexing theory and episodic memory: Updating the index. Hippocampus 2007, 17:1158-1169.

doi:10.1186/1471-2202-12-S1-P142

Cite this article as: Knoblauch and Hauser: STDP, Hebbian cell assemblies, and temporal coding by spike synchronization. $B M C$ Neuroscience 2011 12(Suppl 1):P142.

\section{Submit your next manuscript to BioMed Central} and take full advantage of:

- Convenient online submission

- Thorough peer review

- No space constraints or color figure charges

- Immediate publication on acceptance

- Inclusion in PubMed, CAS, Scopus and Google Scholar

- Research which is freely available for redistribution

Submit your manuscript at www.biomedcentral.com/submit 